\title{
Melioidosis Vaccines: A Systematic Review and Appraisal of the Potential to Exploit Biodefense Vaccines for Public Health Purposes
}

\author{
Sharon J. Peacock ${ }^{1,2,39}$, Direk Limmathurotsakul ${ }^{2,4 * 9}$, Yoel Lubell ${ }^{2}$, Gavin C. K. W. Koh ${ }^{2,3}$, Lisa J. White ${ }^{2}$, \\ Nicholas P. J. Day ${ }^{2,5}$, Richard W. Titball ${ }^{6}$
}

1 Department of Microbiology and Immunology, Faculty of Tropical Medicine, Mahidol University, Bangkok, Thailand, 2 Mahidol-Oxford Tropical Medicine Research Unit, Faculty of Tropical Medicine, Mahidol University, Bangkok, Thailand, 3 Department of Medicine, Cambridge University, Addenbrooke's Hospital, Cambridge, United Kingdom, 4 Department of Tropical Hygiene, Faculty of Tropical Medicine, Mahidol University, Bangkok, Thailand, 5 Nuffield Department of Clinical Medicine, Centre for Clinical Vaccinology and Tropical Medicine, University of Oxford, Churchill Hospital, Oxford, United Kingdom, 6 College of Life and Environmental Sciences, University of Exeter, Exeter, United Kingdom

\begin{abstract}
Background: Burkholderia pseudomallei is a Category B select agent and the cause of melioidosis. Research funding for vaccine development has largely considered protection within the biothreat context, but the resulting vaccines could be applicable to populations who are at risk of naturally acquired melioidosis. Here, we discuss target populations for vaccination, consider the cost-benefit of different vaccination strategies and review potential vaccine candidates.

Methods and Findings: Melioidosis is highly endemic in Thailand and northern Australia, where a biodefense vaccine might be adopted for public health purposes. A cost-effectiveness analysis model was developed, which showed that a vaccine could be a cost-effective intervention in Thailand, particularly if used in high-risk populations such as diabetics. Costeffectiveness was observed in a model in which only partial immunity was assumed. The review systematically summarized all melioidosis vaccine candidates and studies in animal models that had evaluated their protectiveness. Possible candidates included live attenuated, whole cell killed, sub-unit, plasmid DNA and dendritic cell vaccines. Live attenuated vaccines were not considered favorably because of possible reversion to virulence and hypothetical risk of latent infection, while the other candidates need further development and evaluation. Melioidosis is acquired by skin inoculation, inhalation and ingestion, but routes of animal inoculation in most published studies to date do not reflect all of this. We found a lack of studies using diabetic models, which will be central to any evaluation of a melioidosis vaccine for natural infection since diabetes is the most important risk factor.
\end{abstract}

Conclusion: Vaccines could represent one strand of a public health initiative to reduce the global incidence of melioidosis.

Citation: Peacock SJ, Limmathurotsakul D, Lubell Y, Koh GCKW, White LJ, et al. (2012) Melioidosis Vaccines: A Systematic Review and Appraisal of the Potential to Exploit Biodefense Vaccines for Public Health Purposes. PLoS Negl Trop Dis 6(1): e1488. doi:10.1371/journal.pntd.0001488

Editor: Bart J. Currie, Charles Darwin University, Australia

Received October 6, 2011; Accepted December 9, 2011; Published January 31, 2012

Copyright: ( $) 2012$ Peacock et al. This is an open-access article distributed under the terms of the Creative Commons Attribution License, which permits unrestricted use, distribution, and reproduction in any medium, provided the original author and source are credited.

Funding: The study was funded by the Wellcome Trust.S.J.P. is supported by the NIHR Cambridge Biomedical Research Centre. G.C.K.W.K. is supported by a Wellcome Trust Clinical Research Training Fellowship. The funders had no role in study design, data collection and analysis, decision to publish, or preparation of the manuscript.

Competing Interests: The authors have declared that no competing interests exist.

*E-mail: direk@tropmedres.ac

9 These authors contributed equally to this work.

\section{Introduction}

Burkholderia pseudomallei has been the subject of intensive research over the past decade following its classification by the CDC as a category B select agent [1]. The potential for this bacterium to cause clinical disease (melioidosis) after inhalation, coupled with the low infective dose by this route and the ease with which the bacterium can be obtained and cultured are characteristics of a pathogen that might be used for malevolent purposes. Much recent research on $B$. pseudomallei has focused on identifying ways in which the bacterium causes disease with a view to devising biodefense vaccines, and significant progress has been made in demonstrating the feasibility of immunization against melioidosis in animal models [2].
Unlike many of the other biothreat organisms, B. pseudomallei is also an important cause of naturally acquired human infection. This organism is present in the environment across much of SE Asia and $\mathrm{N}$ Australia, and infection results from bacterial inoculation, inhalation or ingestion. Most cases are reported from NE Thailand and N Australia, although melioidosis has been increasingly reported in the Indian subcontinent, China, the Middle East, Africa and South America [3,4]. In NE Thailand, melioidosis is the third most common cause of death from infectious diseases, exceeded only by HIV and tuberculosis [5]. In Darwin, N Australia, melioidosis is the commonest cause of fatal community-acquired septicemic pneumonia [6]. B. pseudomallei is inherently resistant to many antibiotics, including first, second and third generation cephalosporins, aminoglycosides, penicillins and 


\section{Author Summary}

The designation of Burkholderia pseudomallei as a category $B$ select agent has resulted in considerable research funding to develop a protective vaccine. This bacterium also causes a naturally occurring disease (melioidosis), an important cause of death in many countries including Thailand and Australia. In this study, we explored whether a vaccine could be used to provide protection from melioidosis. An economic evaluation based on its use in Thailand indicated that a vaccine could be a cost-effective intervention if used in high-risk populations such as diabetics and those with chronic kidney or lung disease. A literature search of vaccine studies in animal models identified the current candidates, but noted that models failed to take account of the common routes of infection in natural melioidosis and major risk factors for infection, primarily diabetes. This review highlights important areas for future research if biodefence-driven vaccines are to play a role in reducing the global incidence of melioidosis.

polymyxin [7], making the treatment of melioidosis difficult. The associated mortality rate is $43 \%$ in NE Thailand [5] and $14 \%$ in N Australia [8]. Against this background, there may be an opportunity to use vaccines devised for biodefense purposes for the control of naturally acquired infection. Here, we discuss target populations for vaccination, consider the cost-benefit of different vaccination strategies, and review potential vaccine candidates.

\section{Methods}

\section{Cost-effectiveness analysis of vaccination against melioidosis}

We considered the cost-effectiveness of vaccination against melioidosis in NE Thailand, though our models could be applied to other areas of the world where the disease is endemic. We used a Markov model to estimate cost and quality adjusted life years (QALYs) as patients transition between different health states (Figure 1). Table 1 lists the values used for the variables in the model. Our analysis considered the potential benefits of a melioidosis vaccine to reduce (i) disease incidence, and (ii) mortality with varying degrees of protective efficacy for each of these actions. The protective efficacy $(\mathrm{PE})$ and protective duration (PD) of the vaccine were assumed to be homogenous for all routes of disease acquisition. Health benefits were assessed in terms of QALYs gained [9]. The incremental cost-effectiveness ratio (ICER) for a QALY gained was compared with the GDP per capita, which is a proxy measure for the assumed willingness to pay for a QALY gained $[10,11]$. We assumed that Thailand was willing to pay (WTP) \$3,000 for an additional QALY gained which approximates the Thai GDP/capita [10]. Given the uncertainties surrounding the $\mathrm{PE}$ and $\mathrm{PD}$ of a potential vaccine together with its costs and disease incidence in different target populations, results are presented for a broad but plausible range of estimates for each of these parameters. The cost-effectiveness analysis was carried out using Treeage Pro (TreeAge Software Inc., Williamstown, MA, USA).

\section{Search strategy and selection criteria}

We performed a PubMed (MEDLINE) search of the literature using the keywords "pseudomalle", "vaccine", "immunity" and "protect", and reviewed the available references published between January 1911 and Oct 2011. The inclusion criterion used was publication of the vaccine efficacy in humans or in animal models of melioidosis. Initially, titles and abstracts were screened. Articles identified as possibly relevant were reviewed as full text. The reference lists of included articles were assessed for further relevant publications. To inform discussion on the development of vaccine candidates, the animal models used for the vaccine trials were also reviewed.

\section{Results and Discussion}

\section{Target population for a melioidosis vaccine}

Countries that might consider using a biodefense vaccine for the control of endemic melioidosis include Thailand and Australia, where the most reliable estimates of the incidence of melioidosis are available [4]. The annual incidence of human melioidosis in NE Thailand and the Top End of N Australia are currently 21.0 and 19.6 per 100,000 population, respectively [5,12]. However, incidence rates are not uniform within the population, with certain populations at particularly high risk, including those with diabetes mellitus, chronic lung disease or chronic kidney failure [5,12]. For example, the annual incidence rates in diabetics have been estimated to be 145.7 and 260.4 per 100,000 population in $\mathrm{NE}$ Thailand and $\mathrm{N}$ Australia, respectively $[5,12]$. The annual incidence rates in patients in $\mathrm{N}$ Australia with chronic lung disease or chronic kidney disease have been estimated to be 102.0 and 119.6 per 100,000 population, respectively [12]. People older than 35 years are also at higher risk (26.2 versus 4.3 per 100,000 population per year for over or under 35 years of age, respectively) [5].

High-risk groups could be considered as primary targets for melioidosis vaccine trials. One disadvantage may be that generating protective immunity in individuals with such underlying diseases may be difficult to achieve. Furthermore, limiting vaccination to people with diabetes, chronic lung disease or chronic kidney failure would only capture around $60 \%$ to $70 \%$ of all melioidosis cases [5,12]. In addition, around $15 \%$ of patients presenting with melioidosis have previously undiagnosed diabetes. An alternative target group would be all people over 35 years of age residing in an area where melioidosis is known to occur. Vaccinating this much larger group would be predicted to be less cost-effective, but would capture the majority of possible melioidosis patients.

\section{Routes of B. pseudomallei infection and implications for vaccine development}

The commonest routes of $B$. pseudomallei infection are thought to be inoculation, inhalation and ingestion [13]. The prevailing assumption is that most naturally occurring disease results from percutaneous inoculation [13]. This is largely based on the observation that people at high risk such as agricultural workers do not wear protective clothing, work with bare feet, and suffer repeated minor injuries. In addition, disease incidence increases during the rainy season when rice farmers have regular and prolonged contact with contaminated soil and water $[8,14]$. Although entirely feasible, this association is not supported by published evidence. A retrospective study performed in $\mathrm{N}$ Australia found that less than one quarter of people presenting with melioidosis recalled an injury in the preceding weeks [6], and a case-control study conducted in the same setting found that exposure to soil was not associated with melioidosis [15]. Inhalation of B. pseudomallei suspended in aerosols generated from the environment was considered to be an important mechanism for infection in US combatants during the conflict with Vietnam, particularly in helicopter crewmen [16]. Published evidence for inhalation as a route of infection in the general population is 
Table 1. Data used in the cost-effectiveness model.

\begin{tabular}{|c|c|c|}
\hline Inputs & Value & Sources \\
\hline Incidence of melioidosis in NE Thailand & & [5] \\
\hline - In the general population & 21.0 per 100,000 person yrs & \\
\hline - In people older than 35 years old & 26.2 per 100,000 person yrs & \\
\hline - In people with diabetes & 145.7 per 100,000 person yrs & \\
\hline Mortality rate of melioidosis in NE Thailand & $43 \%$ & [5] \\
\hline Population of NE Thailand & 21.4 million & [5] \\
\hline Willingness to pay for a quality-adjusted life year (QALY) gained in Thailand & $\$ 3,000$ & [10] \\
\hline Parameters & Range of parameters & \\
\hline \multicolumn{3}{|l|}{ Vaccine efficacy } \\
\hline - Reduction in incidence of melioiodosis & 0 to $100 \%$ reduction & \\
\hline - Reduction in mortality from melioidosis & 0 to $100 \%$ reduction & \\
\hline Protective duration & 1 to 15 years & \\
\hline Cost of vaccine & $\$ 1$ to $\$ 50$ & \\
\hline
\end{tabular}

limited to several studies from $\mathrm{N}$ Australia that reported a shift towards a higher frequency of pneumonia and severe disease during the rainy season or following heavy monsoon rains and winds $[17,18]$. There is also evidence for ingestion as an important route of $B$. pseudomallei infection. Several clusters of melioidosis cases have been reported from Australia in which a strain of $B$. pseudomallei isolated from a common water source was a genetic match for the strain causing disease in the cluster $[19,20]$. The probability of this occurring by chance is small since $B$. pseudomallei is genetically extremely diverse [21]. B. pseudomallei has also been isolated from public water supplies in 11 locations in the Northern Territory of Australia, genotyped and implicated as a source of infection in 6 locations [22]. In addition, acute suppurative parotitis, which is common in pediatric melioidosis patients in NE Thailand, is presumed to result from direct entry of organisms present in the mouth. In the absence of information on the relative importance of each route of infection it is clear that a melioidosis vaccine for public health purposes should protect against oral, inhalational and percutaneous challenges.

\section{Cost-effectiveness of vaccination against melioidosis}

The model used was based on the effect of a full course of vaccine, which could be either a single inoculation or multiple inoculations. Two target populations were considered: (i) all individuals older than 35 years of age; or (ii) a high-risk group with diabetes mellitus, chronic lung disease or chronic kidney failure. We considered situations where protective efficacy (PE) of a vaccine course ranged from 0 to $100 \%$, protective duration (PD) ranged from 1 to 15 years, and the cost of a full vaccine course ranged from $\$ 1$ to $\$ 50$ (Table 1). PE was assumed to be homogenous across the target population.

Figure 2 shows some situations where a vaccine would be predicted to be cost-effective. With a PD of one year, a vaccine was only cost-effective in the group with major risk factors (estimated annual incidence of 150 per 100,000 persons) and provided that the PE was over $50 \%$ and cost was less than $\$ 2$. If the PD was 3 years, a vaccine at the same price and PE would be cost effective in all adults with an average incidence of 25 per 100,000 . If the PD was 3 years, PE was $100 \%$, and only high risk group were targeted, the full vaccine course could rise to $\$ 10$, and still represent a cost-effective intervention. A vaccine course that reduced both incidence rate and mortality of melioidosis by $50 \%$ with a PD of at least 10 years could cost up to $\$ 10$ in the general adult population and over $\$ 25$ in diabetics and maintain its costeffectiveness.

The potential number of deaths averted and costs associated with different vaccine target populations in NE Thailand assuming that the PE of a vaccine was $50 \%$ are shown in Table 2. The estimates are for a single cycle, and so both the costs and potential number of deaths averted would repeat themselves at the end of the duration of PE.

\section{Vaccine candidates}

We identified 29 studies that examined the following vaccine types: live attenuated $(n=11)$, whole cell killed $(n=5)$, subunit $(\mathrm{n}=9)$, plasmid DNA $(\mathrm{n}=2)$ and dendritic cell $(\mathrm{n}=2)$ (Figure 3). All of the vaccine candidates were evaluated in mouse models, but the $B$. pseudomallei strains used, doses and routes of lethal challenge were highly variable (Table S1). Sterile immunity was rarely reported. Vaccines being developed for biodefence purposes would need to protect primarily against an inhalation challenge. The available experimental evidence indicated that this might be challenging, since the protective efficacy of tested vaccines was greater against intraperitoneal challenge compared with inhalation or intranasal challenge (Table S1). Studies of protection following an ingestion challenge have not been reported.

\section{Live attenuated vaccines}

A wide range of attenuated $B$. pseudomallei mutants have been reported, and immunization of mice with some of these has resulted in the induction of protective immunity [23,24,25,26,27, $28,29,30,31,32,33]$. Live attenuated mutants of $B$. pseudomallei have been shown to be capable of inducing protection against either an injected or an intranasal challenge, but protection was strongly dependent on immunization by the same route as challenge (Table $\mathrm{S} 1)$. A potential advantage of a live attenuated vaccine is the likely ability to induce long-term protection against disease [34]. For example, the live attenuated tularemia vaccine induces cellmediated responses which persist for at least 3 decades [35] and immunity after vaccination with vaccinia virus persists for decades [36] and possibly for the lifetime of the individual [37]. A live 
Table 2. Cost and deaths averted if a melioidosis vaccine ${ }^{a}$ was implemented in NE Thailand.

\begin{tabular}{lllll}
\hline Target group & Population in NE Thailand & Protective duration & Deaths prevented & Cost (single cycle) \\
\hline people older than 35 years old & $\sim 9.5$ million & 1 year & 612 & $\$ 48$ million \\
& & 3 years & 1838 & \\
& & 10 years & 6127 & $\$ 1.3$ million \\
people with diabetes & $\sim 250,000$ & 1 year & 121 & 363 \\
& & 3 years & 1209 & \\
\hline
\end{tabular}

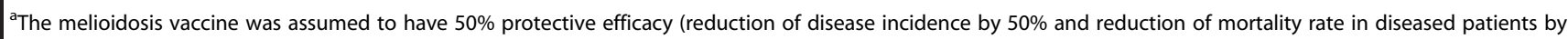
$50 \%$ ) and cost 5 dollars.

doi:10.1371/journal.pntd.0001488.t002

attenuated vaccine against melioidosis that induced long-term protection is likely to be highly cost-effective. However, it may prove difficult to license a live attenuated mutant for use in humans in endemic areas. This bacterium has the potential to cause a potentially life-threatening disease that is difficult to treat, and one would need confidence that reversion of an attenuated mutant to virulence was not possible. In addition, there is a concern that an attenuated mutant might become established as a latent infection. This is found on observation that B. pseudomallei can survive for extend periods in the human host, the longest reported duration of naturally acquired latency prior to clinical symptoms being 62 years [38]. Concerns over the use of a live attenuated meliodosis vaccine are heightened by the knowledge that most naturally occurring cases of disease occur in individuals who are likely to have some degree of immune dysfunction.

\section{Inactivated whole cell vaccines}

Inactivated vaccines are used widely to protect against viral infections, but there are few examples of inactivated bacterial vaccines in current use. Inactivated vaccines are relatively easy and cheap to produce and are capable of inducing protective immunity that persists for several years. For example, protective immunity after immunization with inactivated Salmonella enterica serovar Typhi is reported to persist for at least 30 months $[39,40]$. A potential advantage of killed cell vaccines is their ability to present a wide range of antigens to the immune system. This might be important when considering the genetic and immunological diversity of $B$. pseudomallei. Kill $B$. pseudomallei has resulted in the induction of protective immunity $[41,42,43]$. Killed $B$. thailandensis, a closely related but avirulent organism, was able to induce comparable protection against killed B. pseudomallei [44], and intranasal inoculation of killed B. pseudomallei plus adjuvant CLDC (cationic lipid-DNA complex) gave protection from lethal pulmonary challenge [45]. The main disadvantage of killed cell vaccines is the potential for short-term but undesirable side effects. In other killed cell vaccines, these side effects are largely attributed to the pyrogenic effects of the lipid A portion of lipopolysaccharide (LPS) [46]. However, B. pseudomallei LPS is reported to be at least ten times less potent in eliciting nitric oxide and tumor necrosis factor $\alpha$ from macrophages than LPS from Escherichia coli or Salmonella enterica [47,48]. In addition, B. pseudomallei LPS is less potent than enterobacterial LPS in the induction of pyrogenic activity in rabbits and lethality in galactosamine-sensitized mice [49]. Thus some of the concerns over the use of killed cell vaccines for melioidosis may not be justified, although detailed studies with a killed cell vaccine would be required to confirm the safety and lack of reactogenicity of such a preparation in animal models and humans.

\section{Sub-unit vaccines}

Sub-unit vaccines incorporate specific molecules derived from a microorganism and are the aspiration of most vaccine research programmes. The potential advantages of these vaccines lie with their increased safety and ability to evoke immune responses only to the protective antigen rather than to the entire microorganism. The duration of protection elicited after immunization with a subunit vaccine may differ markedly from vaccine to vaccine and between different population. For example, polysaccharide vaccines often elicit short-lived responses, especially in infants [50]. However, many protein-based vaccines, such as tetanus toxoid and hepatitis $\mathrm{B}$ vaccine, can elicit protective immunity which persists for at least a decade [51,52].

A range of proteins and polysaccharides have been identified that induce different degrees of protection against an intraperitoneal $B$. pseudomallei challenge in mice $[53,54,55,56,57,58,59,60,61]$. Of these, the most protective appear to be LPS, capsular polysaccharide (CPS), LolC protein (an inner membrane protein which forms part of a lipoprotein export system), an outer membrane protein Omp85, and Hcp2 (integral surface-associated component of T6SS). A minority of B. pseudomallei strains produce LPS with an immunologically distinct $\mathrm{O}$-antigen [62], raising the possibility that LPS may not induce protective immunity against all strains. There are no reports of the ability of these individual sub-units to induce significant protection against an inhalation challenge, which would be essential for a biodefense vaccine. However, immunization of mice with outer membrane vesicles, which are likely to contain a combination of sub-units, can provide protection against a low-level (5 $\mathrm{LD}_{50}$ doses) inhalation challenge [61]. There is accumulating evidence, therefore, that subunit vaccines devised for biodefence use may be suitable for use in populations in melioidosis endemic areas.

\section{Naked DNA and dendritic cell vaccines}

There are two reports on the protection afforded by immunisation with DNA vaccines encoding the $B$. pseudomallei flagellar subunit gene, fli $[63,64]$. These showed that immunization of mice with these constructs provided modest levels of protection. However, in general naked DNA vaccines against infectious diseases have been weakly immunogenic in humans even though they have promoted vigorous and effective immune responses in mice [65]. To overcome these limitations prime-boost strategies have often been adopted to allow the development of protective immunity [65]. Two studies have been published on dendritic cells pulsed with heat-killed whole cell $B$. pseudomallei $[66,67]$. Dendritic cell vaccines have been increasingly evaluated in clinical trials for cancer [68], and there is still a need to undertake more proof of principle studies evaluating their utility as vaccines for infectious diseases [69]. 


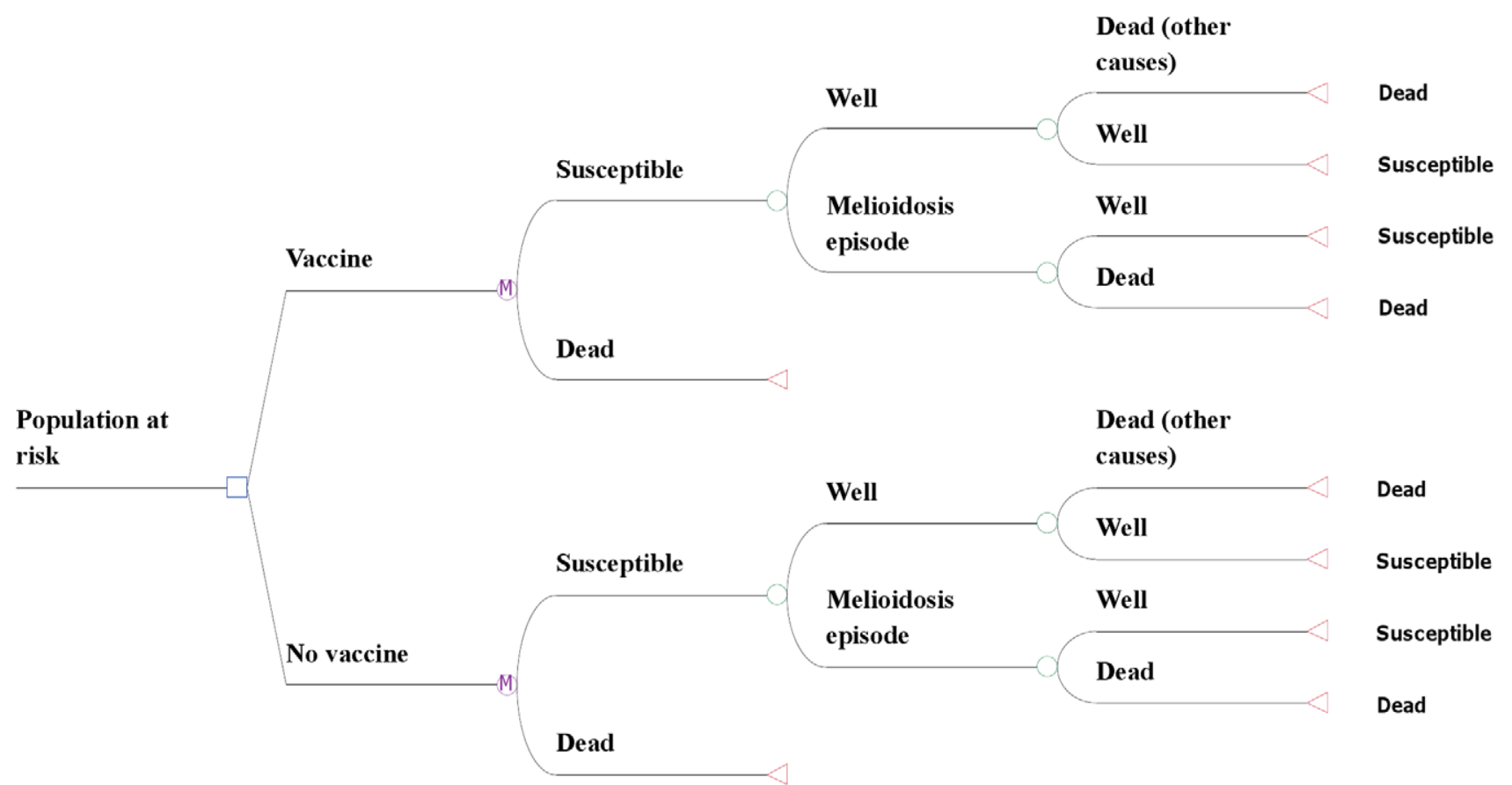

Figure 1. The Markov model used to assess costs and health gains for a melioidosis vaccine. $M$ denotes a Markov node where individuals can transition into the subsequent states in each monthly cycle. In each cycle a susceptible patient can be infected and develop a melioidosis episode, from which they can recover and return to the susceptible state in the next cycle, or die. Patients can also die from natural causes according to their age specific mortality rates.

doi:10.1371/journal.pntd.0001488.g001

\section{Animal models}

Suitable animal models of infection will be central to any melioidosis vaccine development programme. A myriad of different infection models are available, reflecting the different forms of disease in humans. Acute disease occurs in some mouse strains (e.g. BALB/c), and in hamsters with death typically occurring within 7 days of challenge by the intraperitoneal route. Challenge of $\mathrm{BALB} / \mathrm{c}$ mice by the inhalation or intranasal route results in hyperacute disease, with death within a few days. C57BL/6 mice are typically $10^{4}$-fold more resistant to disease than

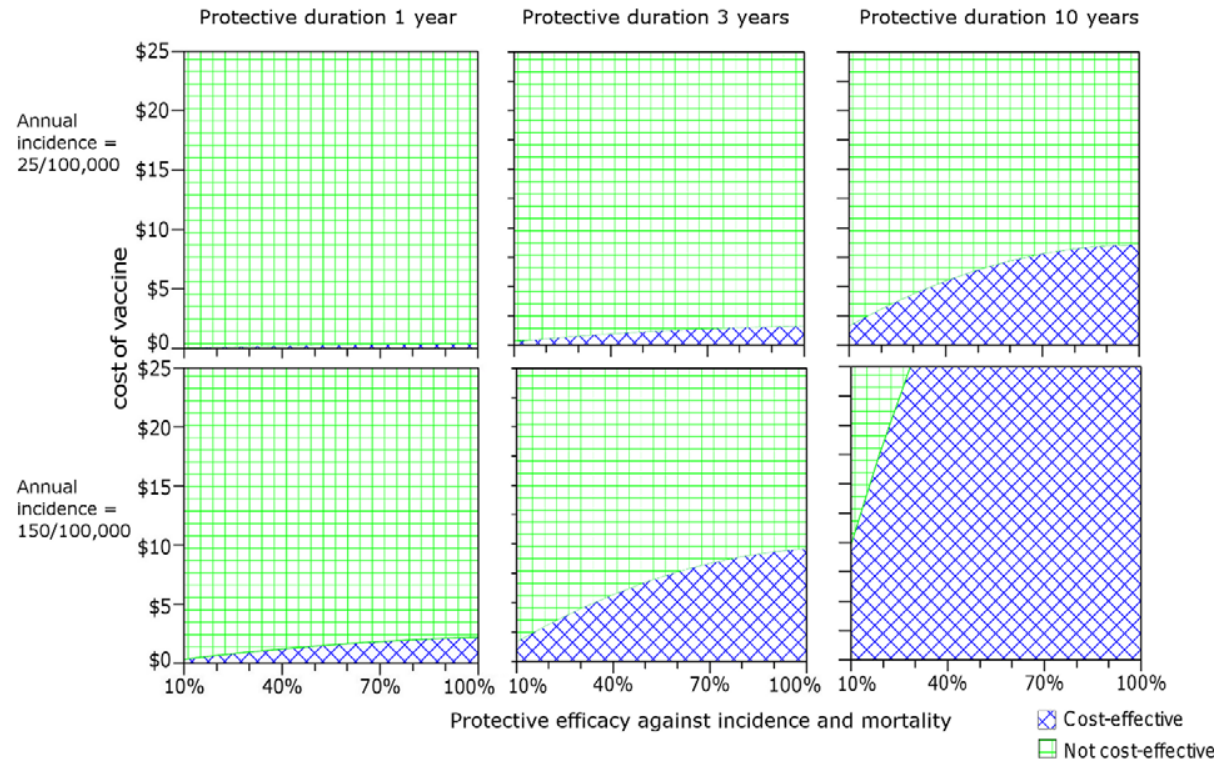

Figure 2. Cost-effectiveness of melioidosis vaccines as determined by incidence, cost, protective efficacy and protective duration. It was assumed that policy makers in Thailand were willing to pay $\$ 3,000$ for an additional QALY gained. Areas in blue indicate where the vaccine is considered cost-effective in the Thai context. The protective efficacy (PE) and protective duration (PD) of the vaccine were considered as homogenous for all routes of disease acquisition. Percentage reduction of PE in this figure was considered as a combination of reduction in both disease incidence and mortality rate.

doi:10.1371/journal.pntd.0001488.g002 
$\mathrm{BALB} / \mathrm{c}$ mice [31], and are more likely to develop a chronic form of the disease which shows similarities with chronic melioidosis in humans [70]. Chronic melioidosis can also be observed in BALB/ c mice after low-dose exposure [71,72]. There are two reports of disease after enteral challenge of mice with B. pseudomallei [73], but there is a clear need for the further development of animal models of disease following ingestion of the bacteria.

Diabetic mouse models of melioidosis have not been well described [74,75], but are highly desirable for evaluating vaccines for natural infection. Mice can be made diabetic for extended periods of time by dosing with streptozotocin, and dendritic cells and macrophages isolated from streptozotocin-induced diabetic mice have altered abilities to ingest and kill B. pseudomallei [76]. Young diabetic rats were found to be susceptible to B. pseudomallei, but not adult diabetic rats $[74,75]$, which is not comparable to the natural situation in humans in which diabetics with melioidosis are predominantly adults.

Efficacy studies in non-human primates are also likely to be required for approval of melioidosis vaccine in human clinical trials. Although mouse models may be useful to screen vaccine candidates, the patterns of disease and immune responses are often different from those seen in human. A range of different non- human primate infection models including marmoset and macaque are currently being developed, but there are currently no reported data on the suitability of these models for testing vaccine candidates.

The form of the disease may also be dependent on the infecting strain. B. pseudomallei is a genetically diverse genus, and there are significant differences in the virulence of different strains of $B$. pseudomallei, at least in BALB/c mice. There is some evidence of genetic variation in strains from different continents, suggesting that it will be important to test vaccines with strains typical of those encountered by the target population. The finding that some strains produce an atypical and immunologically distinct Oantigen means that it may be desirable to test these strains in efficacy studies. However, the low frequency with which naturally occurring disease caused by these strains is encountered also indicates that such testing could be relatively limited. It is of undoubted importance, however, to identify a panel of $B$. pseudomalle $i$ isolates that are representative of the genetic diversity of the bacterium in the range of countries in which the vaccine will be used. Vaccine efficacy should then be evaluated using this test panel with the same standard dosages and multiple routes of inoculation.

\section{2 studies identified through database searching}

\section{3 studies excluded on the basis of title or abstract}

\section{0 articles excluded}

\section{9 studies included in the review}

Figure 3. Study flow diagram.

doi:10.1371/journal.pntd.0001488.g003 


\section{Concluding comments}

We have demonstrated that within a plausible range of estimates for cost and efficacy, it is possible that a vaccine would be cost-effective for the prevention of naturally acquired melioidosis. In Thailand, a vaccine is more likely to be a costeffective intervention if used in high-risk populations such as diabetics and possibly in other at-risk groups such as those working in rice fields. In countries with a higher GDP, it might be cost effective to immunize the entire population in endemic areas as well as tourists or military personnel deploying to melioidosis endemic areas. The finding that a range of candidate vaccines achieved partial protection against disease in mice suggests potential for human use. Reduction in disease severity alone would be predicted to improve outcome in view of the high mortality rate and the fact that half of all in-patient deaths in Thailand occur within the first 48 hours as a result of septic shock. Additional research is required to investigate the potential synergies between vaccination, early antimicrobial treatment and improved care of sepsis in resource-restricted settings. Further studies are also required to determine whether immunization with the best vaccine candidates protects against multiple routes of

\section{References}

1. Dance DAB (2002) Melioidosis. Curr Opin Infect Dis 15: 127-132.

2. Sarkar-Tyson M, Titball RW (2010) Progress toward development of vaccines against melioidosis: A review. Clin Ther 32: 1437-1445.

3. Currie BJ, Dance DA, Cheng AC (2008) The global distribution of Burkholderia pseudomallei and melioidosis: an update. Trans R Soc Trop Med Hyg 102 Suppl 1: S1-4.

4. Limmathurotsakul D, Peacock SJ (2011) Melioidosis: a clinical overview. Br Med Bull 99: 125-39.

5. Limmathurotsakul D, Wongratanacheewin S, Teerawattanasook N, Wongsuvan G, Chaisuksant S, et al. (2010) Increasing incidence of human melioidosis in Northeast Thailand. Am J Trop Med Hyg 82: 1113-1117.

6. Currie BJ, Fisher DA, Howard DM, Burrow JN, Selvanayagam S, et al. (2000) The epidemiology of melioidosis in Australia and Papua New Guinea. Acta Trop 74: 121-127.

7. Wuthiekanun V, Peacock SJ (2006) Management of melioidosis. Expert Rev Anti Infect Ther 4: 445-455.

8. Currie BJ, Ward L, Cheng AC (2010) The epidemiology and clinical spectrum of melioidosis: 540 cases from the 20 year Darwin prospective study. PLoS Negl Trop Dis 4: e900.

9. Rasanen P, Roine E, Sintonen H, Semberg-Konttinen V, Ryynanen OP, et al. (2006) Use of quality-adjusted life years for the estimation of effectiveness of health care: A systematic literature review. Int J Technol Assess Health Care 22: 235-241.

10. Sachs J (2002) Macroeconomics and health: Investing in health for economic development. Geneva: World Health Organization.

11. Shillcutt SD, Walker DG, Goodman CA, Mills AJ (2009) Cost effectiveness in low- and middle-income countries: a review of the debates surrounding decision rules. Pharmacoeconomics 27: 903-917.

12. Currie BJ, Jacups SP, Cheng AC, Fisher DA, Anstey NM, et al. (2004) Melioidosis epidemiology and risk factors from a prospective whole-population study in northern Australia. Trop Med Int Health 9: 1167-1174.

13. Cheng AC, Currie BJ (2005) Melioidosis: epidemiology, pathophysiology, and management. Clin Microbiol Rev 18: 383-416.

14. Suputtamongkol Y, Hall AJ, Dance DA, Chaowagul W, Rajchanuvong A, et al. (1994) The epidemiology of melioidosis in Ubon Ratchatani, northeast Thailand. Int J Epidemiol 23: 1082-1090.

15. Merianos A, Patel M, Lane JM, Noonan CN, Sharrock D, et al. (1993) The 1990-1991 outbreak of melioidosis in the Northern Territory of Australia: epidemiology and environmental studies. Southeast Asian J Trop Med Public Health 24: 425-435.

16. Howe C, Sampath A, Spotnitz M (1971) The pseudomallei group: a review. J Infect Dis 124: $598-606$.

17. Cheng AC, Jacups SP, Ward L, Currie BJ (2008) Melioidosis and Aboriginal seasons in northern Australia. Trans R Soc Trop Med Hyg 102 Suppl 1: S26-29.

18. Currie BJ, Jacups SP (2003) Intensity of rainfall and severity of melioidosis, Australia. Emerg Infect Dis 9: 1538-1542.

19. Inglis TJ, Garrow SC, Henderson M, Clair A, Sampson J, et al. (2000) Burkholderia pseudomallei traced to water treatment plant in Australia. Emerg Infect Dis 6: $56-59$.

20. Currie BJ, Mayo M, Anstey NM, Donohoe P, Haase A, et al. (2001) A cluster of melioidosis cases from an endemic region is clonal and is linked to the water disease acquisition, is effective in diabetic patients, and not accompanied by unacceptable side effects.

\section{Supporting Information}

Table S1 Studies of melioidosis vaccines in animal models.

(DOC)

Checklist S1 PRISMA checklist.

(DOC)

\section{Acknowledgments}

We gratefully acknowledge the support provided by the staff at the Mahidol-Oxford Tropical Medicine Research Unit.

\section{Author Contributions}

Conceived and designed the experiments: SJP DL YL GCKWK LJW NPJD RWT. Analyzed the data: DL YL LJW. Wrote the paper: SJP DL YL GCKWK LJW NPJD RWT. supply using molecular typing of Burkholderia pseudomallei isolates. Am J Trop Med Hyg 65: 177-179.

21. Chantratita N, Wuthiekanun V, Limmathurotsakul D, Vesaratchavest M, Thanwisai A, et al. (2008) Genetic diversity and microevolution of Burkholderia pseudomallei in the environment. PLoS Negl Trop Dis 2: e182.

22. Inglis TJ, Foster NF, Gal D, Powell K, Mayo M, et al. (2004) Preliminary report on the northern Australian melioidosis environmental surveillance project. Epidemiol Infect 132: 813-820.

23. Atkins T, Prior R, Mack K, Russell P, Nelson M, et al. (2002) Characterisation of an acapsular mutant of Burkholderia pseudomallei identified by signature tagged mutagenesis. J Med Microbiol 51: 539-547.

24. Atkins T, Prior RG, Mack K, Russell P, Nelson M, et al. (2002) A mutant of Burkholderia pseudomallei, auxotrophic in the branched chain amino acid biosynthetic pathway, is attenuated and protective in a murine model of melioidosis. Infect Immun 70: 5290-5294.

25. Haque A, Chu K, Easton A, Stevens MP, Galyov EE, et al. (2006) A live experimental vaccine against Burkholderia pseudomallei elicits CD4+ T cellmediated immunity, priming $\mathrm{T}$ cells specific for 2 type III secretion system proteins. J Infect Dis 194: 1241-1248.

26. Stevens MP, Haque A, Atkins T, Hill J, Wood MW, et al. (2004) Attenuated virulence and protective efficacy of a Burkholderia pseudomallei bsa type III secretion mutant in murine models of melioidosis. Microbiology 150: 2669-2676.

27. Rodrigues F, Sarkar-Tyson M, Harding SV, Sim SH, Chua HH, et al. (2006) Global map of growth-regulated gene expression in Burkholderia pseudomallei, the causative agent of melioidosis. J Bacteriol 188: 8178-8188.

28. Cuccui J, Easton A, Chu KK, Bancroft GJ, Oyston PC, et al. (2007) Development of signature-tagged mutagenesis in Burkholderia pseudomallei to identify genes important in survival and pathogenesis. Infect Immun 75: 1186-1195.

29. Breitbach K, Kohler J, Steinmetz I (2008) Induction of protective immunity against Burkholderia pseudomallei using attenuated mutants with defects in the intracellular life cycle. Trans R Soc Trop Med Hyg 102 Suppl 1: S89-94.

30. Srilunchang T, Proungvitaya T, Wongratanacheewin S, Strugnell R, Homchampa P (2009) Construction and characterization of an unmarked aroC deletion mutant of Burkholderia pseudomallei strain A2. Southeast Asian J Trop Med Public Health 40: 123-130.

31. Ulett GC, Labrooy JT, Currie BJ, Barnes JL, Ketheesan N (2005) A model of immunity to Burkholderia pseudomallei: unique responses following immunization and acute lethal infection. Microbes Infect 7: 1263-1275.

32. Norris MH, Propst KL, Kang Y, Dow SW, Schweizer HP, et al. (2011) The Burkholderia pseudomallei Delta asd mutant exhibits attenuated intracellular infectivity and imparts protection against acute inhalation melioidosis in mice. Infect Immun 79: 4010-4018.

33. Easton A, Haque A, Chu K, Patel N, Lukaszewski RA, et al. (2011) Combining vaccination and postexposure $\mathrm{CpG}$ therapy provides optimal protection against lethal sepsis in a biodefense model of human melioidosis. The Journal of infectious diseases 204: 636-644.

34. Vidor E (2010) Evaluation of the persistence of vaccine-induced protection with human vaccines. J Comp Pathol 142 Suppl 1: S96-101.

35. Eneslatt K, Rietz C, Ryden P, Stoven S, House RV, et al. (2011) Persistence of cell-mediated immunity three decades after vaccination with the live vaccine strain of Francisella tularensis. Eur J Immunol 41: 974-980. 
36. Taub DD, Ershler WB, Janowski M, Artz A, Key ML, et al. (2008) Immunity from smallpox vaccine persists for decades: a longitudinal study. Am J Med 121: 1058-1064.

37. Nishiura H, Schwehm M, Eichner M (2006) Still protected against smallpox? Estimation of the duration of vaccine-induced immunity against smallpox. Epidemiology 17: 576-581.

38. Ngauy V, Lemeshev Y, Sadkowski L, Crawford G (2005) Cutaneous melioidosis in a man who was taken as a prisoner of war by the Japanese during World War II. J Clin Microbiol 43: 970-972.

39. Ashcroft MT, Singh B, Nicholson CC, Ritchie JM, Sorryan E, et al. (1967) A seven-year field trial of two typhoid vaccines in Guyana. Lancet 2: 1056-1059.

40. Hejfec LB, Salmin LV, Lejtman MZ, Kuz'minova ML, Vasil'eva AV, et al. (1966) A controlled field trial and laboratory study of five typhoid vaccines in the USSR. Bull World Health Organ 34: 321-339.

41. Razak CE, Ismail G, Embim N, Omar O (1986) Protection studies using whole cells and partially purified toxic material (PPTM) of Pseudomonas pseudomallei. Malays Appl Biol 15: 105-111.

42. Barnes JL, Ketheesan N (2007) Development of protective immunity in a murine model of melioidosis is influenced by the source of Burkholderia pseudomallei antigens. Immunol Cell Biol 85: 551-557.

43. Sarkar-Tyson M, Thwaite JE, Harding SV, Smither SJ, Oyston PC, et al. (2007) Polysaccharides and virulence of Burkholderia pseudomallei. J Med Microbiol 56: 1005-1010.

44. Sarkar-Tyson M, Smither SJ, Harding SV, Atkins TP, Titball RW (2009) Protective efficacy of heat-inactivated B. thailandensis, B. mallei or B. pseudomallei against experimental melioidosis and glanders. Vaccine 27: 4447-4451.

45. Henderson A, Propst K, Kedl R, Dow S (2011) Mucosal immunization with liposome-nucleic acid adjuvants generates effective humoral and cellular immunity. Vaccine 29: 5304-5312.

46. Geurtsen J, Steeghs L, Hamstra HJ, Ten Hove J, de Haan A, et al. (2006) Expression of the lipopolysaccharide-modifying enzymes PagP and PagL modulates the endotoxic activity of Bordetella pertussis. Infect Immun 74: 5574-5585.

47. Utaisincharoen P, Tangthawornchaikul N, Kespichayawattana W, Anuntagool N, Chaisuriva P, et al. (2000) Kinetic studies of the production of nitric oxide (NO) and tumour necrosis factor-alpha (TNF-alpha) in macrophages stimulated with Burkholderia pseudomallei endotoxin. Clin Exp Immunol 122: 324-329.

48. West TE, Ernst RK, Jansson-Hutson MJ, Skerrett SJ (2008) Activation of Tolllike receptors by Burkholderia pseudomallei. BMC Immunol 9: 46.

49. Matsuura M, Kawahara K, Ezaki T, Nakano M (1996) Biological activities of lipopolysaccharide of Burkholderia (Pseudomonas) pseudomallei. FEMS Microbiol Lett 137: 79-83.

50. Rezaei N, Siadat SD, Aghamohammadi A, Moin M, Pourpak Z, et al. (2010) Serum bactericidal antibody response 1 year after meningococcal polysaccharide vaccination of patients with common variable immunodeficiency. Clin Vaccine Immunol 17: 524-528.

51. Wu Q, Zhuang GH, Wang XL, Wang LR, Li N, et al. (2011) Antibody levels and immune memory 23 years after primary plasma-derived hepatitis B vaccination: results of a randomized placebo-controlled trial cohort from China where endemicity is high. Vaccine 29: 2302-2307.

52. Amanna IJ, Carlson NE, Slifka MK (2007) Duration of humoral immunity to common viral and vaccine antigens. N Engl J Med 357: 1903-1915.

53. Nelson M, Prior JL, Lever MS, Jones HE, Atkins TP, et al. (2004) Evaluation of lipopolysaccharide and capsular polysaccharide as subunit vaccines against experimental melioidosis. J Med Microbiol 53: 1177-1182.

54. Harland DN, Chu K, Haque A, Nelson M, Walker NJ, et al. (2007) Identification of a LolC homologue in Burkholderia pseudomallei, a novel protective antigen for melioidosis. Infect Immun 75: 4173-4180.

55. Druar C, Yu F, Barnes JL, Okinaka RT, Chantratita N, et al. (2008) Evaluating Burkholderia pseudomallei Bip proteins as vaccines and Bip antibodies as detection agents. FEMS Immunol Med Microbiol 52: 78-87.

56. Hara Y, Mohamed R, Nathan S (2009) Immunogenic Burkholderia pseudomallei outer membrane proteins as potential candidate vaccine targets. PLoS One 4: e6496.
57. Su YC, Wan KL, Mohamed R, Nathan S (2010) Immunization with the recombinant Burkholderia pseudomallei outer membrane protein Omp85 induces protective immunity in mice. Vaccine 28: 5005-5011.

58. Legutki JB, Nelson M, Titball R, Galloway DR, Mateczun A, et al. (2007) Analysis of peptide mimotopes of Burkholderia pseudomallei exopolysaccharide. Vaccine 25: 7796-7805.

59. Burtnick MN, Brett PJ, Harding SV, Ngugi SA, Ribot WJ, et al. (2011) The cluster 1 type VI secretion system is a major virulence determinant in Burkholderia pseudomallei. Infect Immun 79: 1512-1525.

60. Ngugi SA, Ventura VV, Qazi O, Harding SV, Kitto GB, et al. (2010) Lipopolysaccharide from Burkholderia thailandensis E264 provides protection in a murine model of melioidosis. Vaccine 28: 7551-7555.

61. Nieves W, Asakrah S, Qazi O, Brown KA, Kurtz J, et al. (2011) A naturally derived outer-membrane vesicle vaccine protects against lethal pulmonary Burkholderia pseudomallei infection. Vaccine.

62. Anuntagool N, Wuthiekanun V, White NJ, Currie BJ, Sermswan RW, et al. (2006) Lipopolysaccharide heterogeneity among Burkholderia pseudomallei from different geographic and clinical origins. American Journal of Tropical Medicine and Hygiene 74: 348-352.

63. Chen YS, Hsiao YS, Lin HH, Yen CM, Chen SC, et al. (2006) Immunogenicity and anti-Burkholderia pseudomallei activity in Balb/c mice immunized with plasmid DNA encoding flagellin. Vaccine 24: 750-758.

64. Chen YS, Hsiao YS, Lin HH, Liu Y, Chen YL (2006) CpG-modified plasmid DNA encoding flagellin improves immunogenicity and provides protection against Burkholderia pseudomallei infection in BALB/c mice. Infect Immun 74: 1699-1705.

65. Liu MA (1111) DNA vaccines: an historical perspective and view to the future. Immunol Rev 239: 62-84.

66. Healey GD, Elvin SJ, Morton M, Williamson ED (2005) Humoral and cellmediated adaptive immune responses are required for protection against Burkholderia pseudomallei challenge and bacterial clearance postinfection. Infect Immun 73: 5945-5951.

67. Elvin SJ, Healey GD, Westwood A, Knight SC, Eyles JE, et al. (2006) Protection against heterologous Burkholderia pseudomallei strains by dendritic cell immunization. Infect Immun 74: 1706-1711.

68. Murthy V, Moiyadi A, Sawant R, Sarin R (2009) Clinical considerations in developing dendritic cell vaccine based immunotherapy protocols in cancer. Curr Mol Med 9: 725-731.

69. Garcia F, Routy JP (2011) Challenges in dendritic cells-based therapeutic vaccination in HIV-1 infection Workshop in dendritic cell-based vaccine clinical trials in HIV-1. Vaccine 29: 6454-6463.

70. Leakey A, Ulett G, Hirst R (1998) BALB/c and C57BI/6 mice infected with virulent Burkholderia pseudomallei provide contrasting animal models for the acute and chronic forms of human melioidosis. Microb Pathog 24: 269-275.

71. Conejero L, Patel N, de Reynal M, Oberdorf S, Prior J, et al. (2011) Low-dose exposure of C57BL/6 mice to Burkholderia pseudomallei mimics chronic human melioidosis. Am J Pathol 179: 270-280.

72. Barnes JL, Ketheesan N (2005) Route of infection in melioidosis. Emerg Infect Dis 11: 638-639.

73. West TE, Myers ND, Limmathurotsakul D, Liggitt HD, Chantratita N, et al. (2010) Pathogenicity of high-dose enteral inoculation of Burkholderia pseudomallei to mice. Am J Trop Med Hyg 83: 1066-1069.

74. Brett PJ, Woods DE (1996) Structural and immunological characterization of Burkholderia pseudomallei O-polysaccharide-flagellin protein conjugates. Infect Immun 64: 2824-2828.

75. Woods DE, Jones AL, Hill PJ (1993) Interaction of insulin with Pseudomonas pseudomallei. Infect Immun 61: 4045-4050.

76. Williams NL, Morris JL, Rush C, Govan BL, Ketheesan N (2011) Impact of streptozotocin-induced diabetes on functional responses of dendritic cells and macrophages towards Burkholderia pseudomallei. FEMS Immunol Med Microbiol 61: $218-227$. 